\title{
Cryogenic energy storage system coupled with packed-bed cold storage
}

\author{
Pawet Wojcieszak ${ }^{1, *}$, and Ziemowit Malecha ${ }^{1}$ \\ ${ }^{1}$ Wroclaw University of Science and Technology, Department of Cryogenic, Aeronautic and Process \\ Engineering, Wybrzeze Wyspianskiego 27, 50-370 Wroclaw, Poland
}

\begin{abstract}
Cryogenic Energy Storage (CES) systems are able to improve the stability of electrical grids with large shares of intermittent power plants. In CES systems, excess electrical energy can be used in the liquefaction of cryogenic fluids, which may be stored in large cryogenic vessels for long periods of time. When the demand for electricity is high, work is recovered from the cryogen during a power cycle using ambient or waste heat as an upper heat source. Most research is focused on liquid air energy storage (LAES). However, natural gas can also be a promising working fluid for the CES system. This paper presents a natural gas-based CES system, coupled with a low temperature packed bed cold storage unit. The cold, which is stored at a low temperature level, can be used to increase the efficiency of the cryogenic liquefiers. The model for the packed bed in a high grade cold storage unit was implemented and then compared with the experimental data. The impact of cold recycling on the liquefaction yield and efficiency of the cryogenic energy storage system was investigated
\end{abstract}

\section{Introduction}

Increasing the share of renewable energy sources in the production of electricity is one of the main goals of the European energy strategy. Renewables are considered to be intermittent energy sources - the performance of solar and wind power plants rely strongly on the weather. The production of energy by renewables is, therefore, not sufficiently matched with the demand for it. The development of electrical energy storage methods becomes very important, as a proper energy storage system can improve the stability of an electrical grid. Cryogenic energy storage (CES) systems are potential alternatives to existing electrical energy storage technologies [1], such as pumped hydroelectric storage (PHS) systems or compressed air energy storage (CAES) systems. PHS and CAES systems can provide large storage capacities, but their usage is restricted to specific sites (based on topology and geology). Cryogenic energy storage does not have this drawback.

The main principle of CES systems can be summarized as follows: off-peak electrical energy can be used to liquefy cryogenic fluid. Cryogen is stored in the cryogenic vessels until a discharge of the storage system is required. Then, part of the cryogenic exergy is recovered during a power cycle.

\footnotetext{
*Corresponding author: pawel.wojcieszak@pwr.edu.pl
} 
Most available research on CES systems is focused on liquid air energy storage (LAES), as atmospheric air is widely available and the location of the energy storage plant is not restricted. Nevertheless, natural gas can be a promising working fluid for the CES system because of its potentially higher efficiency [2]. It is also widely available in industrial countries, due to developed gas pipeline system. This paper investigates the cryogenic energy storage using natural gas (NG) as working fluid.

\section{Cryogenic energy storage}

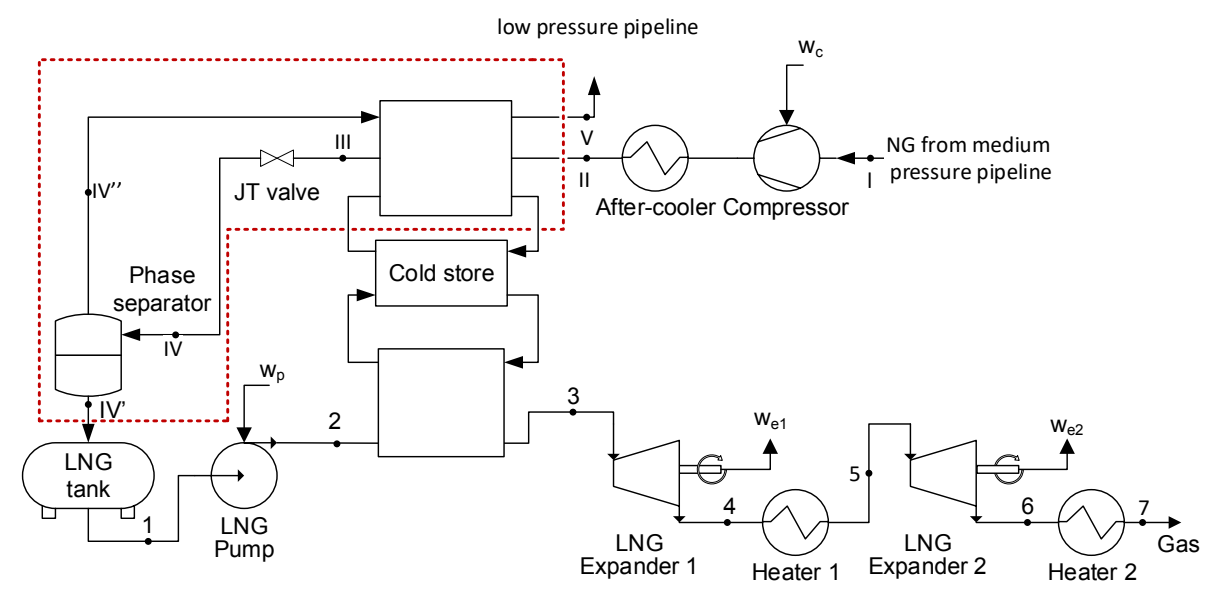

Fig. 1. Cryogenic energy storage system.

The NG-based energy storage system considered in this paper is shown in figure 1. It is based on a simple Linde-Hampson liquefier used for charging, and a direct expansion system with a 2-stage expander that is used for the discharge of energy. Charging and discharging systems are coupled together with the cold storage unit. The proposed system can be placed in the pressure reduction station to lower the compressor work of the liquefier. A purification plant that removes moisture and impurities from the natural gas is not shown in the scheme, but should be installed before liquefaction. Pressure from the medium pressure pipeline (I) is compressed until it reaches high pressure (II), and then it is directed to the recuperative heat exchanger. There, the gas is cooled by vapor return stream (IV') and using the cold stored from the regasification process. Cold, high pressure natural gas (III) is throttled in the JouleThomson valve and part of the stream is liquefied. The liquid (IV') is separated during the separation phase and then directed to the storage vessel. The vapour is then directed through the recuperative heat exchanger to the low pressure gas pipeline. The work of the compressor can be defined as:

$$
w_{c}=T_{a}\left(s_{I}-s_{I I}\right)-\left(h_{I}-h_{I I}\right)
$$

where $T_{a}$ - ambient temperature (K), $s$ - specific entropy $\left(\mathrm{J} \mathrm{kg}^{-1} \mathrm{~K}^{-1}\right), h$ - specific enthalpy $\left(\mathrm{J} \mathrm{kg}^{-1}\right)$. The yield of the liquefaction $(y)$ can be derived from the energy balance (the system boundary was marked with a dashed line in figure 1):

$$
\begin{gathered}
\dot{m} h_{I I}-y \dot{m} h_{I V^{\prime}}-(1-y) \dot{m} h_{V}-\dot{m} q \\
y=\frac{h_{V}-h_{I I}+q}{h_{V}-h_{I V^{\prime}}}
\end{gathered}
$$


where $\dot{m}$ is the mass stream of natural gas at state $\mathrm{I}\left(\mathrm{kg} \mathrm{s}^{-1}\right), q$ is the cooling power delivered per unit of NG mass flow rate $\left(\mathrm{J} \mathrm{kg}^{-1}\right)$. Therefore, work required to liquefy $1 \mathrm{~kg}$ of natural gas can be calculated using formula:

$$
w_{l}=\frac{w_{c}}{y}=\frac{h_{V}-h_{I V^{\prime}}}{h_{V}-h_{I I}+q}\left[T_{a}\left(s_{I}-s_{I I}\right)-\left(h_{I}-h_{I I}\right)\right]
$$

The direct expansion system consists of a pump, a heat exchanger, and expanders (with reheating the natural gas between stages). The LNG from the vessel (1) is pumped until it reaches high pressure (2), and then it is evaporated and heated in the heat exchanger (2-3). The cold storage unit is cooled down for the liquefaction cycle. Warm natural gas at high pressure is then directed to the first expander, where the mechanical power is produced. After the first expander stage, the gas is then reheated and directed to the second expander stage. The specific net work of the system is equal to:

$$
w_{\text {net }}=w_{\text {exp } 1}+w_{\text {exp } 2}-w_{\text {pump }}=\left(h_{3}-h_{4}\right)+\left(h_{5}-h_{6}\right)-\left(h_{2}-h 1\right)
$$

A more detailed description, along with the exergy analysis and optimization of a similar system can be found in [4].

The energy storage efficiency can be defined as a ratio of the specific work recovered during the expansion of a cryogen, to the work required for the liquefaction of $1 \mathrm{~kg}$ of a cryogen:

$$
\eta=\frac{w_{\text {net }}}{w_{l}}
$$

The selected parameters of the energy storage system analyzed in the present study are listed in table 1.

Table 1. Parameters of analysed CES.

\begin{tabular}{|c|c|}
\hline Parameter & Value \\
\hline pressure in medium pressure pipeline & $6 \mathrm{bar}$ \\
\hline pressure in low pressure pipeline & $1.1 \mathrm{bar}$ \\
\hline pump discharge pressure $\left(p_{I I}\right)$ & $100 \mathrm{bar}[4]$ \\
\hline net work of exergy recovery system $\left(w_{\text {net }}\right)$ & $313 \mathrm{~kJ} / \mathrm{kg}[4]$ \\
\hline power output of the exergy recovery system $(P)$ & $350 \mathrm{~kW}$ \\
\hline mass stream of LNG $\left(\dot{m}_{L N G}\right)$ & $1.12 \mathrm{~kg} / \mathrm{s}$ \\
\hline charge (liquefaction) time & $6 \mathrm{~h}$ \\
\hline discharge (expansion) time & $2 \mathrm{~h}$ \\
\hline
\end{tabular}

\section{Cold storage model}

The cold storage unit was assumed to be a packed bed of spherical elements. The parameters of the analyzed packed bed are listed in table 2 . The conceptual sketch of the packed bed thermal storage unit is shown in figure 2. Thermal energy is stored as sensible heat within the material of the packed bed. During a discharge cycle of the CES system, the cooled air in the LNG vaporization process is directed to the bed so that it can be cooled down to the temperature of $140 \mathrm{~K}$. During the liquefaction (charging) cycle of the CES system, warm air flows through the previously cooled bed and is then directed to the recuperative heat exchanger of the liquefier. 


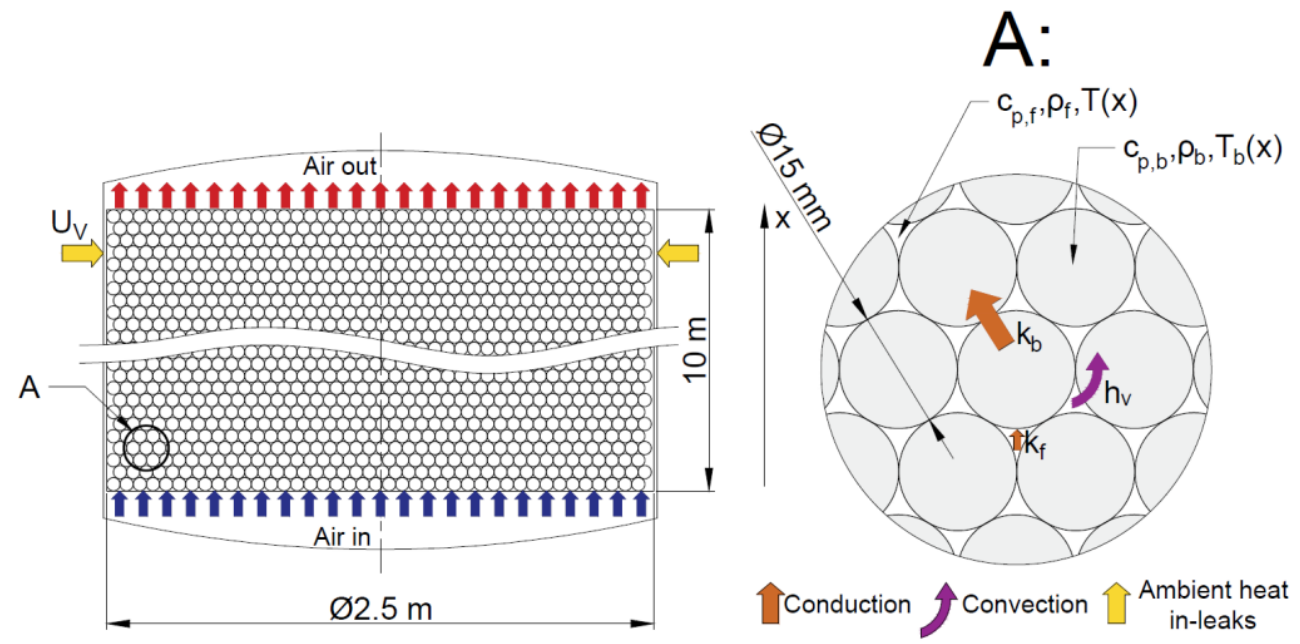

Fig. 2. A conceptual sketch of the packed bed storage that was assumed in the study. Detailed parameters of the packed bed are listed in Table 2 .

Table 2. Parameters of the analysed packed bed storage.

\begin{tabular}{|c|c|}
\hline Parameter & Value \\
\hline diameter $(D)$ & $2.5 \mathrm{~m}$ \\
\hline length $(L)$ & $10 \mathrm{~m}$ \\
\hline storage particle diameter $(d)$ & $15 \mathrm{~mm}$ \\
\hline storage material & quartzite rock \\
\hline void fraction $(\varepsilon)$ & 0.38 \\
\hline working fluid & air $(p=1.5 \mathrm{bar}, T=140-273 \mathrm{~K})$ \\
\hline
\end{tabular}

\subsection{Mathematical model of a cold storage unit}

For the modelling of the packed bed cold storage unit, the following 1-dimensional model was used $[5,6]$ :

$$
\begin{gathered}
\rho_{f} c_{p, f} \frac{\partial T}{\partial t}+\rho_{f} c_{p, f} u_{x} \frac{\partial T}{\partial x}=\frac{\partial}{\partial x}\left(k_{f} \frac{\partial T}{\partial x}\right)+h_{V} \frac{1-\varepsilon}{\varepsilon}\left(T_{b}-T\right) \\
\rho_{b} c_{p, b} \frac{\partial T_{b}}{\partial t}=\frac{\partial}{\partial x}\left(k_{b} \frac{\partial T_{b}}{\partial x}\right)+h_{V}\left(T-T_{b}\right)-U_{v}\left(T_{b}-T_{a}\right)
\end{gathered}
$$

where $\rho$-density $\left(\mathrm{kg} \mathrm{m}^{-3}\right), c_{p}$ - heat capacity $\left(\mathrm{J} \mathrm{kg}^{-1} \mathrm{~K}^{-1}\right), u_{x}$ - velocity $\left(\mathrm{m} \mathrm{s}^{-1}\right), k$ - thermal conductivity, $U_{v}$ - volumetric heat transfer coefficient $\left(\mathrm{W} \mathrm{m}^{-3} \mathrm{~K}^{-1}-\right.$ heat inleaks from an ambient environment). $\varepsilon$ is the void fraction of the bed (ratio of empty volume to the total volume of bed). The subscript $f$ refers to the heat transfer fluid, subscript $b$ - to the solid bed. The convective heat transfer coefficient $h_{v}\left(\mathrm{~W} \mathrm{~m}^{-2} \mathrm{~K}^{-1}\right)$ was obtained using the Colburn factor for gas flowing through the bed of spheres [7], and was calculated using the following formula:

$$
h_{v}=\frac{2.06 R e^{-0.575} G c_{p, f} \cdot 6(1-\varepsilon)}{\varepsilon P r^{2 / 3}}
$$


$G\left(\mathrm{~kg} \mathrm{~m}^{-2} \mathrm{~s}^{-1}\right)$ is the mass flow rate per surface unit, while $R e$ is the Reynolds number, and $\mathrm{Pr}$ - the Prandtl number.

\subsection{Numerical implementation}

The employed mathematical model was implemented in the OpenFOAM (Open Source Field Operation and Manipulation) CFD toolbox $[8,9]$. OpenFOAM is an open-source numerical library for solving partial differential equations (PDEs) in arbitrary geometries, based on the Finite Volume Method. One of the main advantages of OpenFOAM is the possibility to change existing models or to code new models [10].

The diffusion, advection and source terms of the equations $(7,8)$ were discretized implicitly. An explicit discretization of the source term was also considered, for comparison. The implicit treatment of the diffusion terms permitted unconditional stability during the diffusion process, and provided a tridiagonal contribution to the resulting system of algebraic linear equations. Finally, the discretization of the PDE equations $(7,8)$ resulted in the sparse algebraic system, which was solved using the Preconditioned Conjugate Gradient (PCG) method with the Diagonal Incomplete Cholosky (DIC) preconditioner [11]. The residual tolerance of the PCG solver was set to $10^{-6}$ and was satisfied in every time step.

The considered computational domains had lengths of $L=13 \mathrm{~m}$ and $10 \mathrm{~m}$ and were divided into 1000 equispaced computational nodes, resulting in $\mathrm{dx}=0.013 \mathrm{~mm}$ and $0.01 \mathrm{~mm}$, with the time step set to $d t=0.0005 \mathrm{~s}$. The above discretization was checked and confirmed by performing a mesh independence study.

The thermodynamic properties of the heat transfer fluid (air) were obtained using the open-source library CoolProp [12]. To validate the model, the calculations were compared with experimental data available in [6], see figure 3.

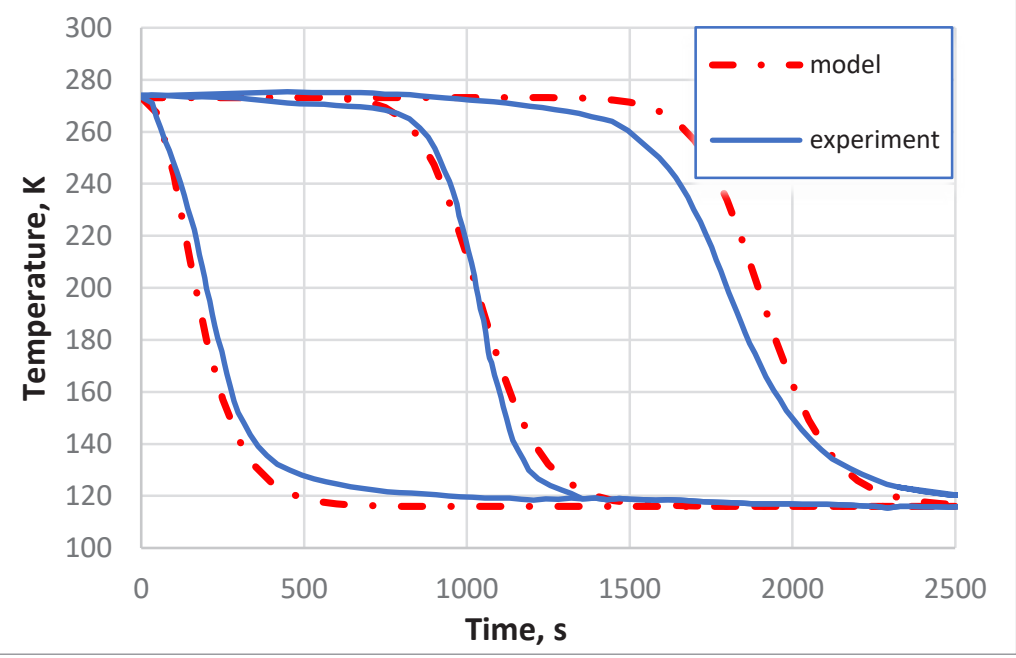

Fig. 3. Validation of cold storage model, using the experimental data provided in [6]. The temperature profiles were obtained for $\mathrm{x}$-coordinates: $0.27,1.7$ and $3.13 \mathrm{~m}$.

\section{Results}

The calculations were initially carried out for the charging process of the bed (the air temperature at the cold storage unit inlet was $140 \mathrm{~K}$ ), and then for the discharge of the cold storage (the air inlet temperature was assumed to be $273 \mathrm{~K}$ ). The temperature profile at the 
beginning of the cold storage discharge process was assumed to be the same as the profile at the end of the charge cycle (the inleaks of heat from the surrounding environment during the time between cycles were neglected). The packed bed temperature profiles at the end of both the charging and discharge cycles are shown in figures 4 and 5. The cold storage was observed to be neither fully charged nor fully discharged.

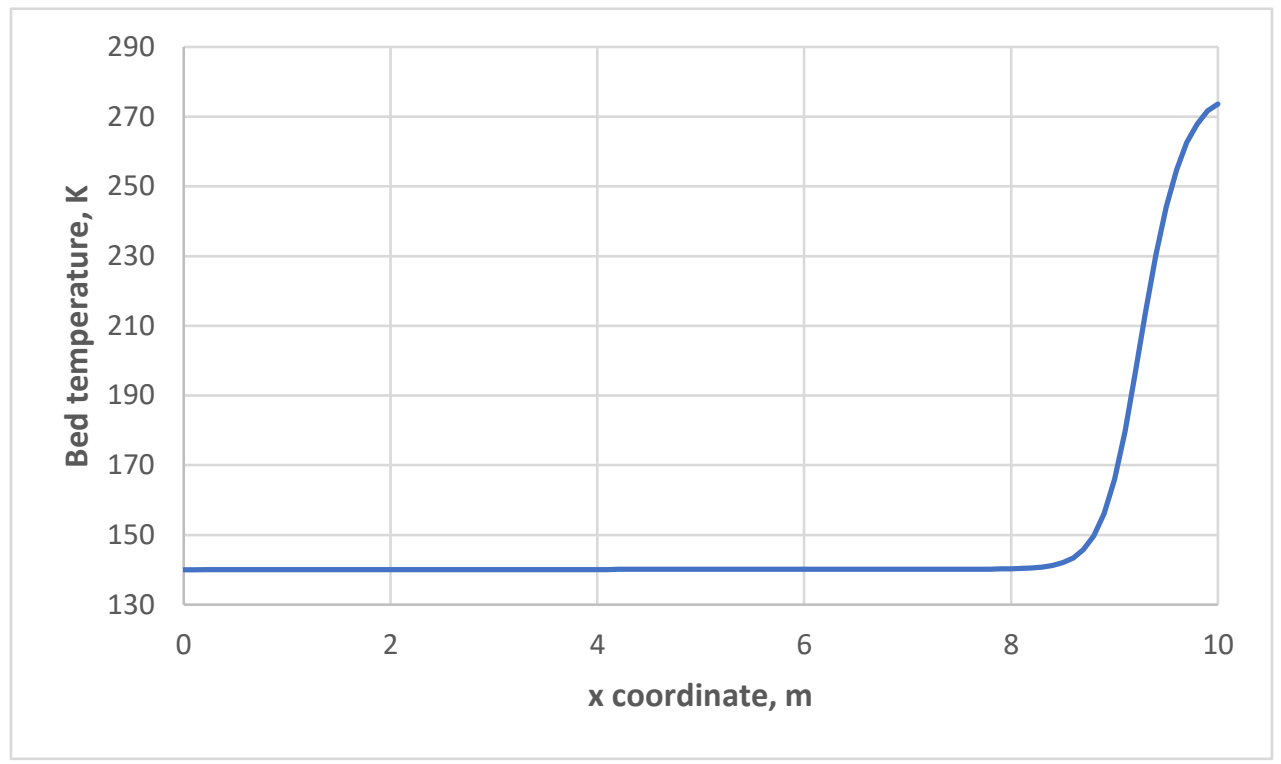

Fig. 4. Bed temperature profile after the cold storage was charged.

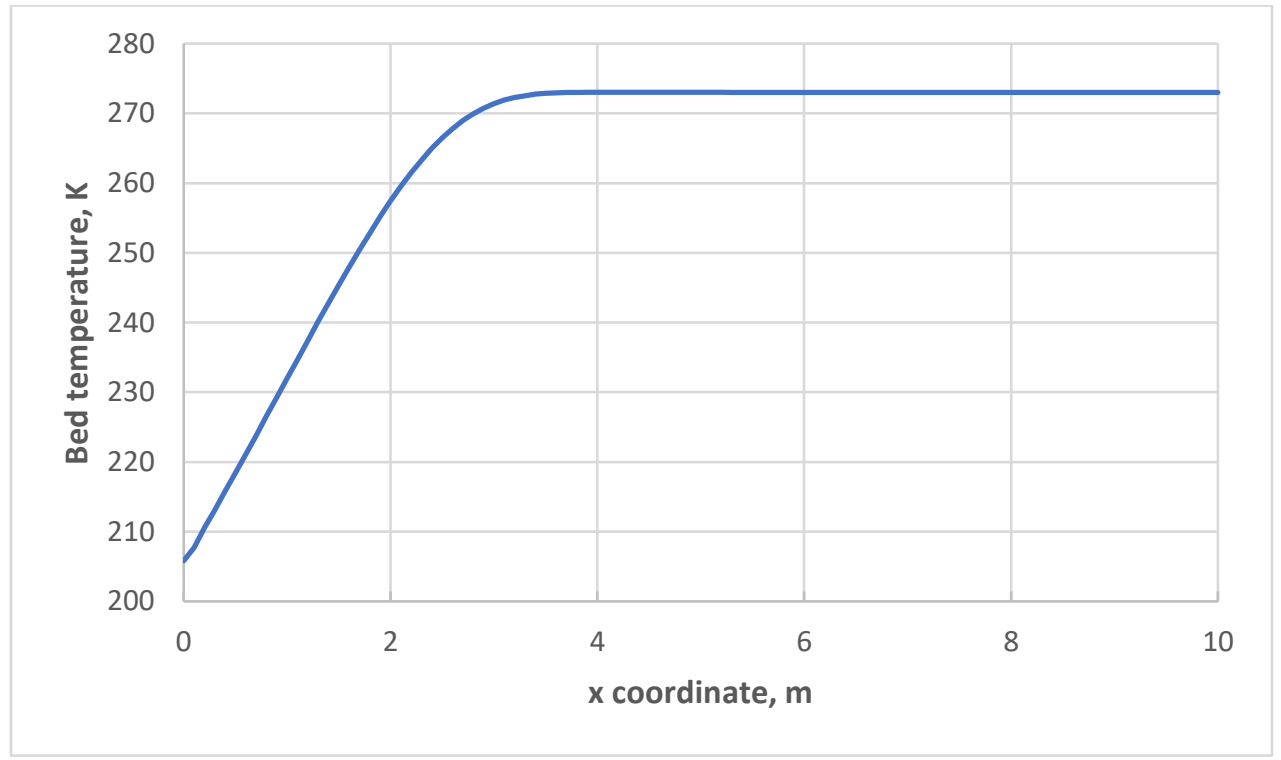

Fig. 5. Bed temperature profile after the discharge of the cold storage.

The cooling power stored in the bed was evaluated using the formula: 


$$
Q_{b e d}=\int_{0}^{L} \rho_{b} c_{p, b} A\left(T_{a}-T_{b}(x)\right) d x
$$

The cooling power used in the liquefaction cycle was assumed to be the difference of the cooling power stored at the beginning and at the end of the cold store discharge cycle. To investigate the influence of cold recycling on the efficiency of a CES system, the liquefaction yield was calculated for the liquefier both with and without the cold recycling.

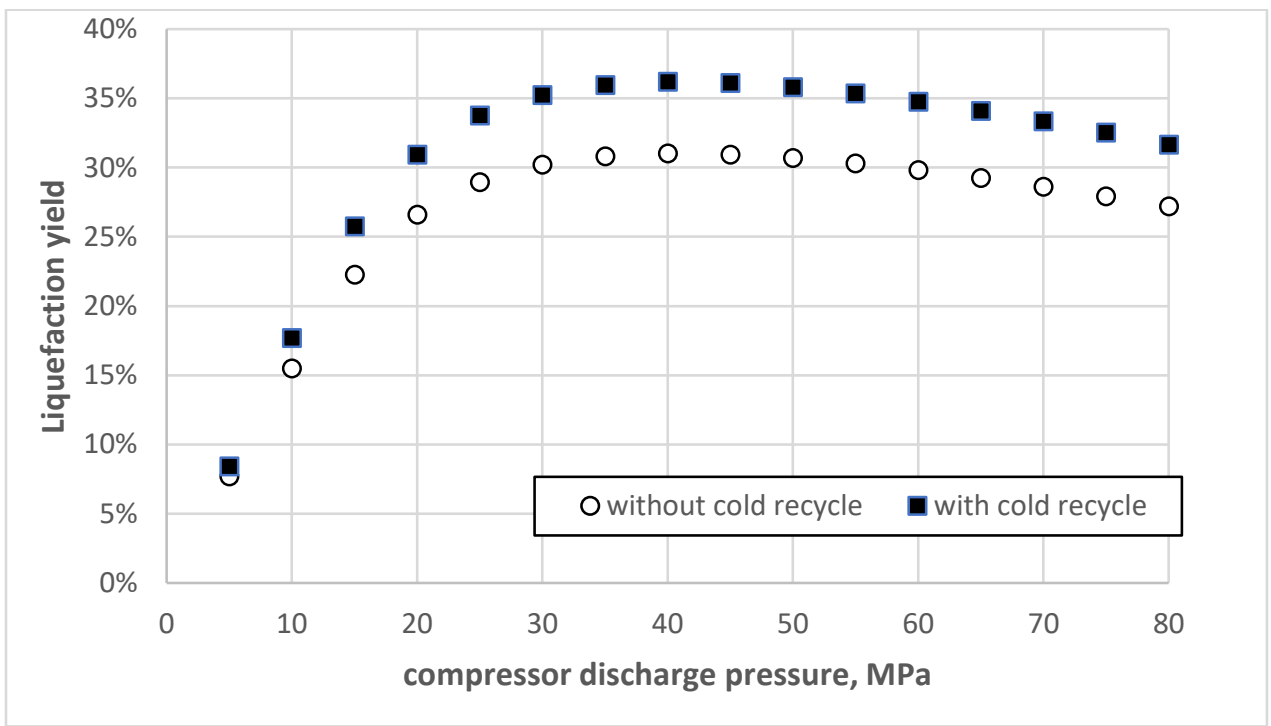

Fig. 6. Influence of cold recycling on the yield of the liquefier.

The minimal work of the liquefaction and energy storage efficiency was also calculated for the CES system, both with and without the cold recycling. The results are presented in table 3 .

Table 2. Comparison of CES systems with and without cold recycle.

\begin{tabular}{|c|c|c|}
\hline Parameter & With cold recycle & Without cold recycle \\
\hline Minimal work of liquefaction & $1383 \mathrm{~kJ} / \mathrm{kg}$ & $1611.6 \mathrm{~kJ} / \mathrm{kg}$ \\
\hline Storage efficiency & $22.6 \%$ & $19.4 \%$ \\
\hline
\end{tabular}

\section{Conclusions}

The usage of cold recycling improved the efficiency of the liquefaction cycle, as well as the efficiency of the entire cryogenic energy storage system. However, it should be noted that the bed was not fully charged and warmed up during the assumed cycles. During the intermittent operation of the system, the charging time of the cold storage unit can be too short to cool down the packed bed. The heat exchange between the warm packed bed and the recuperative heat exchanger of the liquefier can cause a large drop in the liquefaction yield. Therefore, the proper arrangement and operation strategy of the cold storage unit should be further investigated. The concept of a cold storage unit that utilizes latent heat will be further investigated and developed. This will include a modification of the model described by equations $(7,8)$ by incorporating phase change materials (PCM) that can be used for low temperature cold storage. 
The presented work has been undertaken in the framework of the Poland-Taiwan scientific cooperation, as a part of the project "Development of plate heat exchangers for liquid inert gas vaporization, and the modelling of the two-phase flow in heat exchangers" (PHEVAP), financed by The National Center for Research and Development, Poland, No. PL-TWIII/7/2016. The work has been partly supported by statutory funds from Polish Ministry for Science and Higher Education for the year of 2018. Calculations have been carried out using resources provided by the Wroclaw Centre for Networking and Supercomputing (http://wcss.pl), grant No. 460. The authors thank Chia-Luen Lee for her careful proofreading.

\section{References}

1. T.M. Letcher, Storing Energy: With Special Reference to Renewable Energy Sources (Elsevier, Amsterdam, 2016)

2. P. Wojcieszak, J. Poliński, M. Chorowski, IOP Conf. Ser. Mater. Sci. Eng. 278, 012069 (2017)

3. S. Kumar, H.-T. Kwon, K.-H. Choi, W. Lim, J. H. Cho, K. Tak, I. Moon, Appl. Energy 88, 4264 (2011)

4. P. Dorosz, P. Wojcieszak, Z. Malecha, Entropy 20, (2018)

5. M. Cascetta, G. Cau, P. Puddu, F. Serra, Energy Procedia 45, 598 (2014)

6. A. Sciacovelli, A. Vecchi, Y. Ding, Appl. Energy 190, 84 (2017)

7. T.L. Bergman, Introduction to Heat Transfer (Wiley, Hoboken, $6^{\text {th }}$ edition, NJ, 2011)

8. OpenFOAM. The Open Source CFD Toolbox User Guide (The OpenFOAM Foundation, 2009)

9. H.G. Weller, G. Tabor, H. Jasak, C. Fureby, Comput. Phys. 12, 620 (1998)

10. E. Lubryka, Z.M. Malecha, Int. J. Heat Mass Transf. 108, 512 (2017)

11. T.J. Chung, Computational Fluid Dynamics (Cambridge University Press, Cambridge; New York, 2002)

12. I.H. Bell, J. Wronski, S. Quoilin, V. Lemort, Ind. Eng. Chem. Res. 53, 2498 (2014) 